\title{
Comparison between single-beat and multiple-beat methods for estimation of right ventricular contractility
}

\author{
Bernard Lambermont, MD, PhD; Patrick Segers, PhD; Alexandre Ghuysen, MD; Vincent Tchana-Sato, MD; \\ Philippe Morimont, MD; Jean-Michel Dogne, PhD; Philippe Kolh, MD, PhD; Paul Gerard, PhD; \\ Vincent D’Orio, MD, PhD
}

\begin{abstract}
Objective: It was investigated whether pharmacologically induced changes in right ventricular contractility can be detected by a so-called "single-beat" method that does not require preload reduction.

Design: Prospective animal research.

Setting: Laboratory at a large university medical center.

Subjects: Eight anesthetized pigs.

Interventions: End-systolic elastance values obtained by a recently proposed single-beat method $\left(E s_{s b}\right)$ were compared with those obtained using the reference multiple-beat method (Ees $\left.{ }_{m b}\right)$.

Measurements and Main Results: Administration of dobut-
\end{abstract} amine increased Ees $_{\mathrm{mb}}$ from $1.6 \pm 0.3$ to $3.8 \pm 0.5 \mathrm{~mm} \mathrm{Hg} / \mathrm{mL}(p$
$=.001$ ), whereas there was only a trend toward an increase in Ees $_{\text {sb }}$ from $1.5 \pm 0.2$ to $1.7 \pm 0.4 \mathrm{~mm} \mathrm{Hg} / \mathrm{mL}$. Esmolol decreased Ees $_{\mathrm{mb}}$ from $1.7 \pm 0.3$ to $1.1 \pm 0.2 \mathrm{~mm} \mathrm{Hg} / \mathrm{mL}(p=.006)$, whereas there was only a trend for a decrease in Ees $_{\mathrm{sb}}$ from $1.5 \pm 0.2$ to $1.3 \pm 0.1$.

Conclusions: The present method using single-beat estimation to assess right ventricular contractility does not work as expected, since it failed to detect either increases or decreases in right ventricular contractility induced by pharmacologic interventions. (Crit Care Med 2004; 32:1886-1890)

KEY WoRDS: conductance catheter; contractile function; hemodynamics; inotropic agents; right ventricular function
$\mathrm{T}$

he assessment of ventricular contractility is one of the cornerstones of the evaluation of ventricular systolic function (1). The development and validation of the conductance catheter method for the measurement of pressure-volume (PV) loops allowed the determination of indexes of ventricular contractility, first mainly in the left ventricle (2) and later in the right ventricle as well (3). However, determination of PV-based contractile indexes, such as the end-systolic PV or preload recruitable stroke work (PRSW) relationships, requires the recording of several PV loops during a transient preload reduction. Such conditions are usually obtained by caval vein occlusion, in the experimental setting. In hu-

From the Hemodynamic Research Center (BL, AG, VT-S, PM, J-MD, PK, VD) and Department of Statistics $(\mathrm{PG})$, University of Liege, Liege, Belgium; and the Hydraulics Laboratory (PS), Institute Biomedical Technology, Ghent University, Ghent, Belgium.

Supported, in part, by Fonds de la Recherche Scientifique Médicale (VT-S, PK).

Copyright $\odot 2004$ by the Society of Critical Care Medicine and Lippincott Williams \& Wilkins

DOI: 10.1097/01.CCM.0000139607.38497.8A mans, however, this procedure may be hazardous and rises ethical criticism.

To circumvent the necessity of preload reduction, several "single-beat" methods have been proposed for the left ventricle, based on data measured during hemodynamic steady-state conditions. Although the proposed methodologies appear attractive, Kjorstad et al. (4) demonstrated that none of the proposed single-beat methods was able to provide an accurate evaluation of left ventricular contractility.

However, since the physiology and anatomy of the left and right ventricles differ largely, it remains to be shown whether the conclusion of Kjorstad et al. (4), observed for the left ventricle, also applies to the right ventricle.

The aim of our study was therefore to evaluate, for the right ventricle, the original single-beat method based on work of Sunagawa et al. (5) and further elaborated by Takeuchi et al. (6). PV loops recorded by the conductance catheter were used to compare end-systolic elastance values obtained by the single-beat method $\left(\right.$ Ees $_{\mathrm{sb}}$ ) with those obtained using the standard multiple beats method (i.e., the slope $\left[\mathrm{Ees}_{\mathrm{mb}}\right]$ of the end-systolic PV relationship) during pharmacologic interventions known to alter right ventricular contractility.

\section{MATERIALS AND METHODS}

All experimental procedures and protocols used in this investigation were reviewed and approved by the Ethics Committee of the Medical Faculty of the University of Liege. The investigation conforms with the guidelines (7) published by the U.S. National Institutes of Health.

\section{Surgical Preparation}

Experiments were performed on eight healthy Pietran pigs of either gender weighing from 20 to $30 \mathrm{~kg}$. The animals were premedicated with intramuscular administration of ketamine $(20 \mathrm{mg} / \mathrm{kg})$ and diazepam $(1 \mathrm{mg} / \mathrm{kg})$. Anesthesia was then induced and maintained by a continuous infusion of sufentanil $\left(0.5 \mu \mathrm{g} \cdot \mathrm{kg}^{-1} \cdot \mathrm{hr}^{-1}\right)$ and pentobarbital (5 $\left.\mathrm{mg} \cdot \mathrm{kg}^{-1} \cdot \mathrm{hr}^{-1}\right)$. Spontaneous movements were prevented by pancuronium bromide $(0.2$ $\mathrm{mg} / \mathrm{kg}$ ). After endotracheal intubation through a cervical tracheostomy, the pigs were connected to a volume-cycled ventilator (Evita 2, Dräger, Lübeck, Germany) set to deliver a tidal volume of $10 \mathrm{~mL} / \mathrm{kg}$ with an $\mathrm{FIO}_{2}$ of 0.4 and at a respiratory rate of 20 breaths/ 
min. End-tidal $\mathrm{CO}_{2}$ measurements (Capnomac, Datex, Helsinki, Finland) were used to monitor the adequacy of ventilation. Respiratory settings were adjusted to maintain endtidal $\mathrm{CO}_{2}$ between 30 and $35 \mathrm{~mm} \mathrm{Hg}$.

The heart and the pulmonary trunk were exposed by means of medial sternotomy. A micromanometer-tipped catheter (Sentron pressure-measuring catheter, Cordis, Miami, $\mathrm{FL}$ ) was inserted into the main pulmonary artery through a stab wound in the right ventricular outflow tract. A 14-mm diameter perivascular flow probe (Transonic Systems, Ithaca, NY) was closely adjusted around the main pulmonary artery $2 \mathrm{~cm}$ downstream of the pulmonary valve. The micromanometertipped catheter was manipulated so that the pressure sensor was positioned closely to the flow probe.

Left atrial pressure was measured with a micromanometer-tipped catheter inserted into the cavity through the left atrial appendage. Systemic arterial pressure was monitored with a micromanometer-tipped catheter inserted into the descending thoracic aorta through the right femoral artery.

A 7-Fr, 12-electrode ( $8 \mathrm{~mm}$ interelectrode distance) conductance micromanometertipped catheter (CD Leycom, Zoetermeer, The Netherlands) was inserted through the right ventricular infundibulum into the right ventricle and positioned so that all electrodes were in the right ventricular cavity.

A 6-Fr Fogarty balloon catheter (Baxter Healthcare, Oakland, CA) was advanced into the inferior vena cava through a right femoral venotomy. Inflation of this balloon produced a titratable gradual preload reduction.

\section{Experimental Protocol and Physiologic Measurements}

Data were recorded before and during pharmacologic modulation of ventricular contractility using a) esmolol infusion at 1 $\mathrm{mg} \cdot \mathrm{kg}^{-1} \cdot \mathrm{min}^{-1}$ during 5 mins to decrease contractility; and b) dobutamine infusion at $10 \mu \mathrm{g} \cdot \mathrm{kg}^{-1} \cdot \mathrm{min}^{-1}$ during 15 mins to enhance contractility. Esmolol and dobutamine infusions were randomly administered in each animal and separated by a 30-min resting period.

Hemodynamic data included measurement of pulmonary artery pressure (PAP), pulmonary artery blood flow, left atrial pressure, systemic arterial pressure, heart rate, right ventricular pressure, right ventricular volume, and right ventricular PV loops. These variables were recorded during a short apneic phase and stored for subsequent analysis. All analog signals were continuously converted to digital form with an appropriate $\mathrm{AD}$ converter system (Codas, DataQ Instruments, Akron, $\mathrm{OH})$. Systemic arterial pressure, PAP, and pulmonary blood flow waves were integrated to calculate corresponding mean values. Right ventricular PV loops were recorded during transient occlusion of the inferior vena cava using the Fogarty balloon.

At each step, two sets of data were recorded and analyzed.

\section{Data Analysis}

Mulitple-Beat Estimation of Right Ventricular Contractility. Right ventricular PV loops were obtained using the conductance catheter method (3). The application, validation, and calibration of the conductance technique for measuring ventricular volume were described elsewhere (2). Although this technique was originally designed for use in the left ventricle, the feasibility of conductance catheter measurements in the right ventricle has been also demonstrated (3). Briefly, a multiple-electrode catheter placed in the right ventricle is used to set up an electrical field, and adjacent pairs of electrodes measure the local conductivity of blood, which is proportional to local blood volume (3). The instantaneous conductance signals, $\mathrm{G}(\mathrm{t})$, are converted to volume signals, $\mathrm{V}(\mathrm{t})$, owing to the following equation:

$$
\mathrm{V}(\mathrm{t})=(1 / \alpha)\left[L^{2} \cdot \rho \cdot \mathrm{G}(\mathrm{t})-\mathrm{V}_{\mathrm{c}}\right]
$$

where $\alpha$ is a dimensionless gain factor, $L$ the distance between the sensing electrodes, $\rho$ the resistivity of the blood, and $V_{c}$ the correction volume for the conductance of the surrounding tissue, commonly referred to as parallel conductance. Parallel conductance can be estimated by transiently altering the conductivity of blood with hypertonic saline (3). To determine the gain factor $\alpha$, an alternate method of measuring volume is needed (3). In this study, we used the value of stroke volume measured by the pulmonary artery ultrasonic flow probe. Before each measurement, parallel conductance was determined with the saline method by injecting $3 \mathrm{~mL}$ of $\mathrm{NaCl} 10 \%$ into the inferior vena cava (3).

Ees $_{\mathrm{mb}}$ was determined during a rapid inferior vena cava occlusion maneuver by fitting a straight line through the end-systolic PV points (3). During this interval of preload reduction, the slope of the linear relationship between stroke work and end-diastolic volume (PRSW) was calculated. Stroke work was referred to as the integrated area of each PV loop.

Single-Beat Estimation of Right Ventricular Contractility. Ten consecutive cardiac cycles were selected from steady-state portions during each experimental period. The digitized data for each group of measurements were analyzed by a computer-assisted system. Pressure and volume curves for each recording period were averaged to produce mean pressure and mean volume waveforms, in order to eliminate any noise artifact and compensate for beat-to-beat variations. Such obtained mean ventricular pressure and volume waves were then graphically opposed to gen- erate a PV loop representative of each experimental period. Individual cycles were identified using the onset of ventricular contraction, time-aligned and numerically averaged. The left top corner of the PV loop determined the end-systolic point (Pes, Ves), whereas the right-bottom corner of the PV loop determined the end-diastolic volume (Ved). The method consisted of fitting ventricular pressure during the isovolumic contraction and relaxation periods to a sinusoidal function, given as

$$
\mathrm{P}_{\text {sine }}(\mathrm{t})=\mathrm{A}+\mathrm{B} \sin (\mathrm{Ct}+\mathrm{D})
$$

where A through D are variables obtained from the fitting procedure, which was based on minimizing the square difference between measured and fitted pressure during the isovolumic periods. The onset of the isovolumic contraction period was taken as the point where right ventricular change in pressure in time $(\mathrm{dP} / \mathrm{dt})$ reached $10 \%$ of peak positive $\mathrm{dP} /$ $\mathrm{dt}$, whereas the end of the isovolumic contraction period was taken as the moment of peak positive $\mathrm{dP} / \mathrm{dt}$. Similarly, the onset of isovolumic relaxation was taken as the moment of peak negative $\mathrm{dP} / \mathrm{dt}$, whereas the end of this period was assumed to be the point where $\mathrm{dP} / \mathrm{dt}$ reached $10 \%$ of peak negative $\mathrm{dP} / \mathrm{dt}$. $\mathrm{P}_{\max }$ was the maximal value of $\mathrm{P}_{\text {sine }}$ and an estimate of the pressure that would be generated by the ventricle in an isovolumic beat at Ved (Fig. 1).

Single-beat elastance, $\mathrm{Ees}_{\mathrm{sb}}$, is then taken as the slope of the line connecting two points in the PV plane: (Ved, $\mathrm{P}_{\max }$ ) and (Ves, Pes), and is thus given by $\operatorname{Ees}_{\mathrm{sb}}=\left(\mathrm{P}_{\max }-\mathrm{Pes}\right) /(\mathrm{Ved}-$ Ves).

\section{Statistical Analysis}

Data are presented as mean \pm SEM. Statistical comparison of data before and after each intervention was conducted by a paired Student's $t$-test (Statistica, Statsoft, Tulsa, OK). Comparison of multiple-beat and single-beat Ees estimation was obtained by applying analysis of agreement (8). We considered $p<.05$ to be statistically significant.

\section{RESULTS}

Two sets of data were collected in the eight pigs during each of the four experimental conditions, that is, before and after both dobutamine and esmolol infusions. Out of these 64 sets of data, five were finally excluded from analysis due to the difficulty of making sine function fit the pressure curve in three single-beat records, whereas poor quality in two PV loops made it impossible to apply both multiple-beat and single-beat method analysis. 

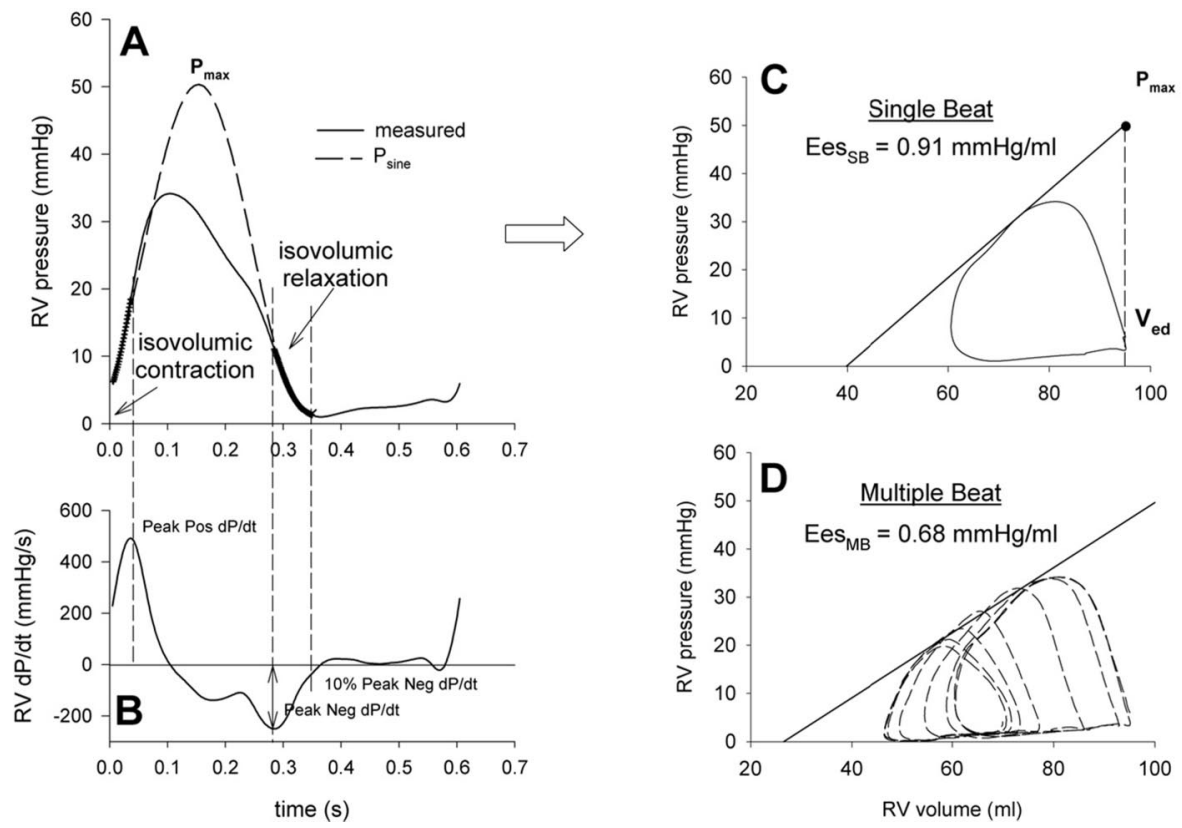

Figure 1. Single-beat method to obtain end-systolic pressure-volume relationship (ESPVR). B, delineation of both isovolumic contraction and isovolumic relaxation time delays is based on right ventricular $(R V)$ change in pressure in time $(d P / d t)$. $A$, a sinusoidal function $\left(P_{\text {sine }}\right)$ is then adjusted to fit isovolumic segments of the ventricular pressure contour. The maximum amplitude of the sine wave is taken as $\mathrm{P}_{\max }$, referred to as the maximum pressure that the ventricle should release if ejection was impeded (nonejecting beat). ESPVR is then plotted as a line defined by two different points whose coordinates are Pmax and Ved (nonejecting beat) and P and V measured at end-systole (ejecting beat), respectively. $D$, as a reference, the corresponding multiple-beat ESPVR computation is provided. $E e s_{s b}$, end-systolic elastance values obtained by the single-beat method; $E_{m b}{ }_{m b}$, end-systolic elastance values obtained by the multiple-beat method.

There was no significant difference between values of Ees $_{\mathrm{mb}}$ and $\mathrm{Ees}_{\mathrm{sb}}(1.7 \pm$ 0.2 and $1.4 \pm 0.1 \mathrm{~mm} \mathrm{Hg} / \mathrm{mL}$, respectively) at the beginning of the experimental period.

\section{Effects of Dobutamine Infusion}

During administration of dobutamine, heart rate increased from $117 \pm 8$ to 142 \pm 7 beats $/$ min $(p<.05)$, mean PAP values increased from $17 \pm 2$ to $20 \pm 2 \mathrm{~mm}$ Hg $(p<.05)$, cardiac output increased from $62 \pm 3$ to $82 \pm 4 \mathrm{~mL} / \mathrm{sec}(p<.05)$, and mean systemic arterial pressure remained unchanged.

Administration of dobutamine increased Ees $_{\mathrm{mb}}$ values from $1.6 \pm 0.3$ to $3.8 \pm 0.5 \mathrm{~mm} \mathrm{Hg} / \mathrm{mL}(p=.001)$, whereas there was only a trend toward an increase in Ees $_{\mathrm{sb}}$ from $1.5 \pm 0.2$ to $1.7 \pm 0.4 \mathrm{~mm}$ $\mathrm{Hg} / \mathrm{mL}(p=.5$; Fig. 2). Administration of dobutamine increased the slope values of PRSW relationships from $27 \pm 6$ to $41 \pm$ $6 \mathrm{~mm} \mathrm{Hg}(p<.05)$.

\section{Effects of Esmolol Infusion}

During administration of esmolol, heart rate decreased from $116 \pm 7$ to 98 \pm 3 beats/min $(p<.05)$, mean PAP values increased from $18 \pm 2$ to $20 \pm 2 \mathrm{~mm}$ Hg $(p<.05)$, cardiac output decreased from $64 \pm 2$ to $50 \pm 2 \mathrm{~mL} / \mathrm{sec}(p<.05)$, and mean systemic arterial pressure decreased from $84 \pm 6$ to $77 \pm 5 \mathrm{~mm} \mathrm{Hg}(p$ $<.05)$.

After administration of esmolol, Ees $_{\mathrm{mb}}$ decreased from $1.7 \pm 0.3$ to $1.1 \pm 0.2$ $\mathrm{mm} \mathrm{Hg} / \mathrm{mL}(p=.006)$, whereas there was only a trend for a decrease in Ees $_{\mathrm{sb}}$ from $1.5 \pm 0.2$ to $1.3 \pm 0.1 \mathrm{~mm} \mathrm{Hg} / \mathrm{mL}$ $(p=.09$; Fig. 3). Administration of esmolol decreased the slope values of PRSW relationships from $25 \pm 4$ to $15 \pm 2 \mathrm{~mm}$ Hg $(p<.05)$.

\section{Multiple-Beat Vs. Single-Beat Estimation of Ees}

Correlation between Ees $_{\mathrm{mb}}$ and Ees $\mathrm{s}_{\mathrm{sb}}$ after pharmacologic interventions is presented in Figure 4. Ees $_{\mathrm{sb}}$ values were also compared with Ees $\mathrm{mb}_{\mathrm{mb}}$ values by application of an analysis of agreement (8). Bias was $0.54 \mathrm{~mm} \mathrm{Hg} / \mathrm{mL}$, and limits of agreement were $\pm 3.4 \mathrm{~mm} \mathrm{Hg} / \mathrm{mL}$ (Fig. 4).
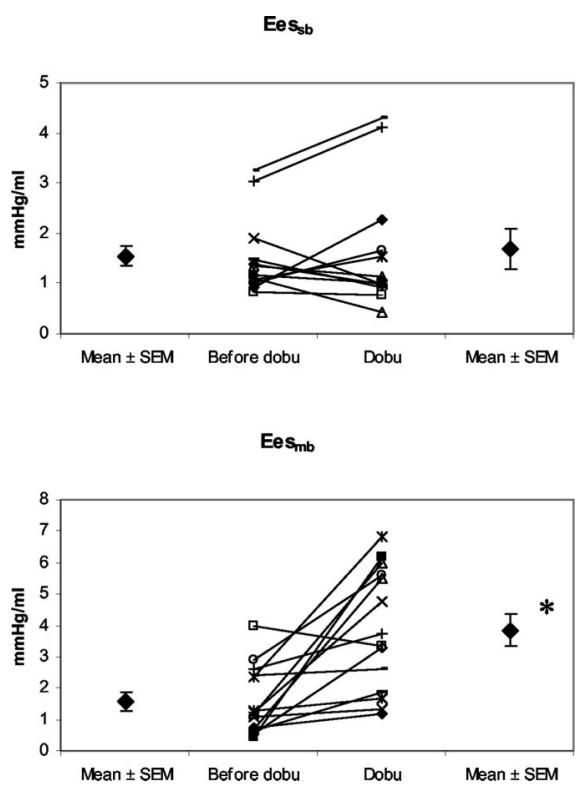

Figure 2. Slope of end-systolic pressure-volume relationship before (baseline) and during dobutamine (Dobu) infusion obtained by single-beat $E e$ $\left.s_{s b}\right)$ and multiple-beat $\left(E e s_{m b}\right)$ methods. ${ }^{*} p<.05$.
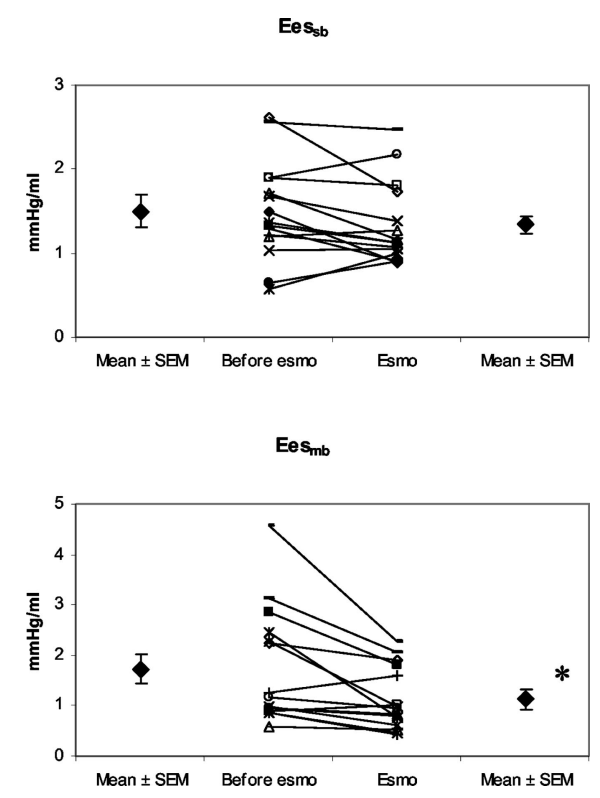

Figure 3. Slope of end-systolic pressure-volume relationship before (baseline) and during esmolol $(E s m o)$ infusion obtained by single-beat $\left(E e s_{s b}\right)$ and multiple-beat $\left(E e_{m b}\right)$ methods. ${ }^{*} p<.05$.

\section{DISCUSSION}

Several single-beat methods have been advanced to assess ventricular contractility without changing ventricular loading conditions. However, none of these variants could provide better results than the original analysis described by Takeuchi et al. (6), selected therefore as the reference single-beat method to evaluate right ven- 

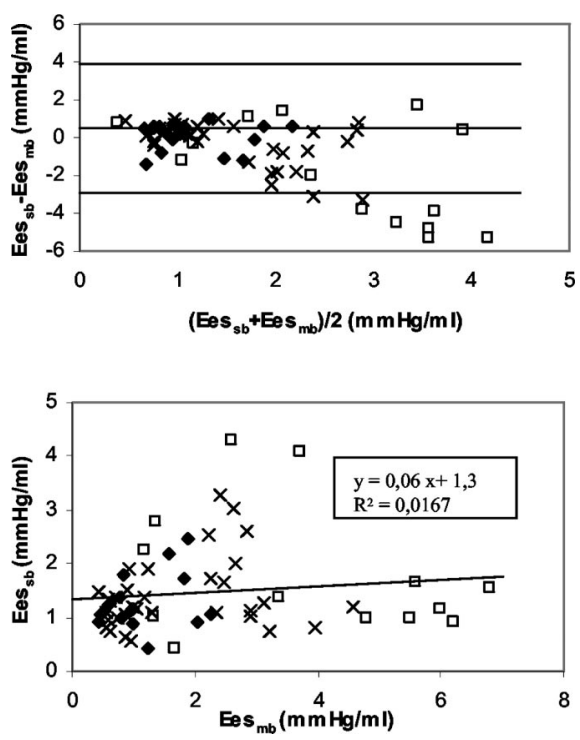

Figure 4. Scatterplots of analysis of agreement between single-beat $\left(\operatorname{Ees}_{s b}\right)$ and multiple-beat $\left(E e s_{m b}\right)$ methods before $(\times)$ and during dobutamine (squares) or esmolol (diamonds) infusions and comparison between Ees $_{\mathrm{sb}}$ and Ees $\mathrm{s}_{\mathrm{mb}}$ before $(X)$ and during dobutamine (squares) or esmolol (diamonds) infusions.

tricular contractility index in the present work. We therefore compared two methods for the evaluation of right ventricular contractility at baseline, during dobutamine and esmolol infusion. These contractile indexes were derived from PV loops recorded by the conductance catheter technique during either steady state (single-beat method) or transient preload reduction (multiple-beat method). Our results indicate that $\operatorname{Ees}_{\mathrm{sb}}$ is less accurate than $\mathrm{Ees}_{\mathrm{mb}}$ in predicting right ventricular contractility changes induced by pharmacologic interventions.

Baseline values of contractility measured in our study were similar to those reported in the literature $(3,9)$. Although there was no difference in mean basal Ees values obtained by the two methods, and although the systematic bias of $0.54 \mathrm{~mm}$ $\mathrm{Hg} / \mathrm{mL}$ was acceptable, limits of agreement $( \pm 3.4 \mathrm{~mm} \mathrm{Hg} / \mathrm{mL})$ between the two methods were unacceptably high. This reflects the random errors of the single-beat method and is directly related to the predictive value of the test.

As expected, Ees mb $_{\text {increased during }}$ dobutamine infusion and decreased during esmolol infusion $(3,9,10)$. In parallel, PRSW relationship slope values were significantly increased by dobutamine infusion, whereas significant reduction occurred after esmolol administration. In contrast, neither dobutamine nor esmo- lol infusions induced significant change in $\mathrm{Ees}_{\mathrm{sb}}$. These results are in accordance with those of Kjorstad et al. (4) for the left ventricle, where none of the applied single beat methods could predict an increase in contractility induced by dopamine. However, right and left ventricular physiology and anatomy differ, and, therefore, the present single-beat method had to be tested specifically for the right ventricle (11).

In recent work, Brimioulle et al. (9) showed that the right ventricular $\mathrm{P}_{\max }$ of a nonejecting beat can be calculated by a single-beat analysis and may subsequently be used to calculate $\operatorname{Ees}_{\mathrm{sb}}$. Ees $_{\mathrm{sb}}$ obtained by such a method could predict changes in right ventricular contractility induced by dobutamine. However, their conclusions were weakened by the absence of comparison between this method and a gold standard. Moreover, PV loops were obtained by integration of the instantaneous pulmonary arterial flow and not by using the conductance catheter technique (12).

The lack of a significant decrease in Ees $_{\mathrm{sb}}$ during esmolol infusion could, in addition to the low sensitivity of the single-beat method, be attributed to the rather modest reduction in contractility induced by esmolol. Using the single-beat method, Brimioulle et al. (9) also found no difference in contractility after propranolol infusion, whereas Dickstein et al. (3) showed a very slight decrease in Ees after esmolol infusion.

Comparison of single-beat and multiple-beat estimation of contractility showed bias and limits of agreement similar to those reported in the literature for the left ventricle (4). It shows the lack of precision of the single-beat method particularly when Ees values are far from physiologic values, as it was the case in a number of animals after administration of dobutamine. In the single-beat method applied in this study, a sine wave is fitted to the ventricular pressure during isovolumic contraction and relaxation. This leads to a number of inherent problems. First, as noted by Kjorstad et al. (4), a sine wave is a rather stiff, symmetrical wave shape. Isovolumic ventricular contraction and relaxation are not necessarily symmetrical, especially in cardiovascular disease or during pharmacologic interventions. Inotropic stimulation leads to specific changes in the shape of ventricular pressure curve compared with the control period. In fact, the isovolumic contraction pressure increase becomes

\section{$\checkmark$ he present method using single-beat estimation to assess}

right ventricular contractility does not work as expected, since it failed to detect either increases or decreases in right ventricular contractility induced by pharmacologic interventions.

considerably steeper than the decrease of the pressure decay during isovolumic relaxation (4). Such a feature implies that maximal pressure developed by the ventricle is necessarily shifted to the left leading to an asymmetry in the shape of the ventricular pressure curve. Such an asymmetry is not accounted for by the sine function used to fit the ventricular curve. As a result, the single-beat computation method leads to an underestimation of end-systolic elastance values during inotropic stimulation since it fails to accurately reflect the leftward-induced shift of maximum ventricular pressure.

Another problem, which mainly applies to the right ventricle, is the fact that the isovolumic periods are rather short, especially the isovolumic relaxation period. This means not only that there is little information available for the fitting procedure, increasing the uncertainty on the fitted curve and $\mathrm{P}_{\max }$, but also, and perhaps even more important, that the method is sensitive to the choice of the onset and end of the isovolumic contraction and relaxation period, respectively. It was not specified by Brimioulle et al. (9) how the end and onset of the isovolumic relaxation periods were determined. Following Leeuwenburgh et al. (13), we selected cutoff values for $\mathrm{dP} / \mathrm{dt}$ (the points where $10 \%$ of peak positive or negative $\mathrm{dP} / \mathrm{dt}$ are reached) to delineate the isovolumic contraction and relaxation periods.

\section{CONCLUSIONS}

To the best of our knowledge, our work is the first one designed to compare 
a single-beat method to the gold standard multiple-beat method for the assessment of right ventricular contractility. The method using single-beat estimation to assess right ventricular contractility does not work as expected, since it failed to detect either increases or decreases in right ventricular contractility induced by pharmacologic interventions. Therefore, single-beat methods still remain an open field of hemodynamic research and need to be further investigated to accurately assess right ventricular contractility.

\section{REFERENCES}

1. Wojtczak JA, Szalados JE: Right heart function: Neither silent nor passive. Crit Care Med 2002; 30:2601-2603

2. Baan J, van der Velde ET, de Bruin HG, et al: Continuous measurement of left ventricular volume in animals and humans by conductance catheter. Circulation 1984; 70 : 812-823
3. Dickstein ML, Yano O, Spotnitz HM, et al: Assessment of right ventricular contractile state with the conductance catheter technique in the pig. Cardiovasc Res 1995; 29: $820-826$

4. Kjorstad KE, Korvald C, Myrmel T: Pressurevolume-based single-beat estimations cannot predict left ventricular contractility in vivo. Am J Physiol Heart Circ Physiol 2002; 282: H1739-H1750

5. Sunagawa K, Yamada A, Senda Y, et al: Estimation of the hydromotive source pressure from ejecting beats of the left ventricle. IEEE Trans Biomed Eng 1980; 27:299-305

6. Takeuchi M, Igarashi $\mathrm{Y}$, Tomimoto $\mathrm{S}$, et al: Single-beat estimation of the slope of the end-systolic pressure-volume relation in the human left ventricle. Circulation 1991; 83: 202-212

7. Guide for the Care and Use of Laboratory Animals (NIH Publication No. 85-23). Bethesda, MD, U.S. National Institutes of Health, 1996

8. Bland JM, Altman DG: Statistical methods for assessing agreement between two meth- ods of clinical measurement. Lancet 1986; 1:307-310

9. Brimioulle S, Wauthy P, Ewalenko P, et al: Single-beat estimation of right ventricular end-systolic pressure-volume relationship. Am J Physiol Heart Circ Physiol 2003; 284: H1625-H1630

10. Vogel M, Schmidt MR, Kristiansen SB, et al: Validation of myocardial acceleration during isovolumic contraction as a novel noninvasive index of right ventricular contractility: Comparison with ventricular pressurevolume relations in an animal model. Circulation 2002; 105:1693-1699

11. Dell'Italia LJ, Santamore WP: Can indices of left ventricular function be applied to the right ventricle? Prog Cardiovasc Dis 1998; 40:309-324

12. Steendijk P: Right ventricular function and failure: Methods, models, and mechanisms. Crit Care Med 2004; 32:1087-1089

13. Leeuwenburgh BP, Steendijk P, Helbing WA, et al: Indexes of diastolic RV function: Load dependence and changes after chronic RV pressure overload in lambs. Am J Physiol Heart Circ Physiol 2002; 282:H1350-H1358 